\title{
Círculos de interaprendizaje para incrementar logro satisfactorio en resolución de problemas de cantidad en estudiantes de primer año de secundaria
}

Circles of interlearning to increase satisfactory achievement in problem solving of first year high school students

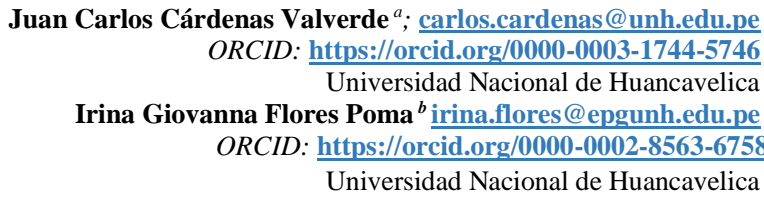

Universidad Nacional de Huancavelica

Recibido: Octubre / 05 /2021 - Revisado: Noviembre /25 /2021 - Publicado: Diciembre /29 / 2021

\section{RESUMEN}

Las instituciones emergentes logran resultados favorables cuando trabajan en equipo; por ello, se planteó el objetivo conocer los efectos que tienen los círculos de interaprendizaje para incrementar logro satisfactorio en la competencia resolución de problemas de cantidad en estudiantes de primer año de secundaria. La investigación es aplicada, de nivel explicativo, con una muestra de 62 estudiantes del primer año de secundaria de una institución educativa de Lima Metropolitana. Las pruebas pedagógicas pasaron por la validez y confiabilidad para ser suministradas. Se empleó la prueba “T” para aceptar la hipótesis alterna, concluyendo que los círculos de interaprendizaje tienen efectos significativos para incrementar "Logro Satisfactorio" en la competencia resolución de problemas en estudiantes de primer año de secundaria.

Palabras claves: Círculos de interaprendizaje, Logro satisfactorio, Resolución de problemas de cantidad, Instituciones emergentes

\begin{abstract}
Emerging institutions achieve favorable results when they work as a team; Therefore, the objective was set to know the effects that interlearning circles have to increase satisfactory achievement in the quantity problem solving competence in first year high school students. The research is applied, at an explanatory level, with a sample of 62 first-year high school students from an educational institution in Metropolitan Lima. The pedagogical tests passed through the validity and reliability to be supplied. The " $T$ " test was used to accept the alternative hypothesis, concluding that the interlearning circles have significant effects to increase "Satisfactory Achievement" in problem solving competence in first year high school students.
\end{abstract}

Keywords: Inter-learning circles, Satisfactory achievement, Quantity problem solving, emerging institutions 


\section{$\underline{\text { Introducción }}$}

La Organización de las Naciones Unidas para la Educación, la Ciencia y la CulturaUNESCO (2019) sostiene que "la educación es uno de los derechos fundamentales de los seres humanos; aporta al logro de su desarrollo integral, promueve su libertad y autonomía, y contribuye al ejercicio pleno de otros derechos humanos". Por lo mencionado, las instituciones educativas emergentes dedican su esfuerzo para continuar sobresaliendo y obtener resultados favorables en beneficio de los estudiantes y docentes. Las estrategias prescindibles en la mejora como institución vienen a ser la aplicación de las comunidades profesionales de aprendizaje, círculos de interaprendizaje y trabajo en equipo.

UNESCO (2019) destaca que "las comunidades profesionales de aprendizaje, tanto en las instituciones educativas (II.EE.) como en las redes educativas, descansan en hábitos de trabajo colaborativo que permiten a los docentes de aula y directivos desarrollar habilidades para interactuar de manera productiva. Ello genera condiciones para asumir la participación en los programas formativos de manera colegiada, promoviendo el aprendizaje conjunto y el uso de lo aprendido para la toma de decisiones sobre la práctica pedagógica”.

En las II.EE. de Lima metropolitana, se aplican estrategias innovadoras para mantener el ranking alcanzado respecto a los logros de aprendizaje; cada área, grado y nivel educativo es responsable de mantener y superar esta expectativa. La experiencia muestra el empleo de círculos de interaprendizaje; a nivel de docentes, pues se viene obteniendo resultados favorables en un trabajo colaborativo y participativo. En la presente investigación, se traslada esta experiencia a nivel de estudiantes. Para ello se formuló el objetivo conocer los efectos que tienen los círculos de interaprendizaje para incrementar logro satisfactorio en la competencia resolución de problemas de cantidad en estudiantes de primer año de secundaria.

Ante este panorama, estudios realizados sobre círculos de interaprendizaje fueron investigados por Vigo Romero (2018) en la tesis cuando "plantea la aspiración de mejorar la escala de resultado en comprensión de textos escritos en los estudiantes de secundaria, aplicándose la guía de entrevista, permitiendo en concordancia con los resultados de la 
evaluación censal y los obtenidos en las actas de evaluación establecer el diagnostico, además teniendo en cuenta las propuestas de Solé en su texto estrategias de lectura. A todo esto, el directivo como líder pedagógico debe mantener actualizados a sus docentes aplicando los círculos de interaprendizaje y un monitoreo, y acompañamiento efectivo para brindar una educación de calidad con resultados significativos en el proceso de enseñanza y aprendizaje en un ambiente acogedor practicando una comunicación asertiva”.

Membrillo Cabrera (2018) sostienen que "la tecnología en los últimos años, ha proporcionado herramientas diversas y útiles, las cuales ayudan a optimizar procesos cotidianos de la vida diaria y del aprendizaje; en este caso, la investigación se centra en el uso de la tecnología como apoyo en el proceso del interaprendizaje colaborativo".

Cornejo Turpo (2018) plantea el objetivo en la tesis sobre "la gestión pedagógica del docente y los grupos de interaprendizaje para ver si existe relación entre la gestión pedagógica del docente y los grupos de interaprendizaje en las instituciones educativas de la ciudad de Juliaca; además, establece la hipótesis con respecto a si existe relación entre la gestión pedagógica del docente y los grupos los grupos de interaprendizaje en las instituciones educativas de la ciudad de Juliaca".

Cabezas García (2016) manifiesta que "los círculos de interaprendizaje tienen efectos positivos bajo el diseño no experimental de tipo transversal-descriptivo simple; la población de estudio estuvo conformada por 100 estudiantes, la muestra seleccionada - también de 100 estudiantes - fue de tipo no probabilística y censal”.

Fuentes López, Páez Gomez, \& Prieto (2019) plantean que "el trabajo colaborativo a través de los círculos de interaprendizaje permite resultados que ayudan a alcanzar objetivos deseados en torno al enfoque mixto cualitativo".

Del mismo modo, investigaciones realizadas sobre resolución de problemas fueron estudiados por Añaños Flores \& Asencios Gonzáles (2018) cuando plantean como objetivo “determinar cómo influye la resolución de problemas en el aprendizaje en los estudiantes del cuarto grado de educación secundaria a través del método Pólya. Este consta de cuatro pasos 
que buscan desarrollar las competencias para actuar y pensar matemáticamente en situaciones de cantidad, regularidad equivalencia y cambio, forma movimiento y localización gestión de datos, e incertidumbre”.

Domínguez Osorio \& Espinoza Santiago (2019) realizaron la investigación con el “objetivo de potenciar la resolución de problemas matemáticos desarrollando habilidades de pensamiento a través de la implementación del método Heurístico en los estudiantes $3^{\circ}$ de primaria básica de la Institución Mundo Bolivariano. A partir de allí, se inicia un proceso tendiente a determinar la incidencia de las variables independientes: el método heurístico y las habilidades de pensamiento sobre la variable dependiente, la resolución de problemas".

Albán Alcívar (2018) en la investigación "la matemática es una disciplina fundamental del plan de estudio en todos los niveles de educación regular. Su aprendizaje es imprescindible para el desarrollo del pensamiento lógico y crítico, e interpretar y resolver problemas matemáticos, cada vez más complejos. Los estudiantes del primer ciclo de la Unidad Académica de Ciencias Agropecuarias, de la Universidad Técnica de Machala, provincia El Oro, Ecuador, presentaron falencias en los procesos de resolución de problemas matemáticos, lo que generó la necesidad de realizar la presente investigación, que tiene como objetivo describir que estrategias emplean los estudiantes y su relación con el rendimiento académico en la solución de problemas".

Finalmente, Paredes Lozada (2019) planteó como objetivo “determinar de qué manera las estrategias metodológicas, para resolver problemas, inciden en el desarrollo de las capacidades matemáticas de los estudiantes del sexto grado de primaria de la Institución Educativa N. 1137 José Antonio Encinas, que corresponde a la jurisdicción de la Unidad de Gestión Educativa Local (UGEL) N. ${ }^{\circ}$ 06, órgano desconcentrado del Ministerio de Educación".

\section{Círculos de interaprendizaje}

Toribio Vargas y Guerrero Ortiz (2017) manifiestan "que los círculos de interaprendizaje son espacios de intercambio de experiencias y aprendizaje para docentes, y 
directivos de la misma escuela o comunidad, en los cuales se abordan grupalmente temas de interés común sobre el trabajo pedagógico. Favorece la socialización y valoración de las prácticas docentes exitosas a través de la discusión y reflexión. Además, permite profundizar en determinadas estrategias pedagógicas. Exige un alto grado de participación de los asistentes. Además, demanda el registro de las ideas fuerzas de los participantes, así como los consensos que deriven de la reflexión".

A su vez, en el Proyecto Regional Andino Perú-Bolivia (2010) encontramos que "Círculo de interaprendizaje es una estrategia educativa intercultural que parte de la comunidad para aprender en colectivo".

UNESCO (2019) sostiene que "en el trabajo colegiado el aprendizaje docente, como ningún otro, tiene una base fuerte en el intercambio de experiencias, en la identificación de problemas comunes y en la búsqueda compartida de soluciones. La experiencia internacional aporta conocimiento sobre el desarrollo profesional docente como un proceso de colaboración, interacciones, aprendizajes compartidos y afirmación del trabajo en equipo en un marco de lineamientos nacionales claros".

El Ministerio de Educación ( $\mathrm{s} / \mathrm{a}$ ) da a conocer "los objetivos del grupo de interaprendizaje, los cuales son los siguientes: dar respuesta de manera pertinente a las necesidades de formación de los docentes; promover la formación de equipos de docentes a través del trabajo cooperativo; reforzar colectivamente la aplicación de la propuesta educativa; y, uso de materiales educativos, estrategias de captación y permanencia de estudiantes en los círculos de aprendizaje”.

Asimismo, el Ministerio de Educación (s/a) divulga las “características de un grupo: interacción recíproca, existencia de objetivos, valores y actividades compartidas, estabilidad y duración relativa, conciencia de grupo, y reconocimiento como tal".

\section{Resolución de problemas de cantidad:}


En el Currículo Nacional, el Ministerio de Educación del Perú (2016) señala que "la competencia resolución de problemas de cantidad consiste en que el estudiante solucione problemas o plantee nuevos problemas que le demanden construir y comprender las nociones de cantidad, número, de sistemas numéricos, sus operaciones y propiedades. Además, dotar de significado a estos conocimientos en la situación y usarlos para representar o reproducir las relaciones entre sus datos y condiciones implica, también, discernir si la solución buscada requiere darse como una estimación o cálculo exacto, y para ello selecciona estrategias, procedimientos, unidades de medida y diversos recursos. El razonamiento lógico en esta competencia es usado cuando el estudiante hace comparaciones, explica a través de analogías, induce propiedades a partir de casos particulares o ejemplos, en el proceso de resolución del problema”.

Ministerio de Educación del Perú (2016) agrega que "esta competencia implica, por parte de los estudiantes, la combinación de las siguientes capacidades: traduce cantidades a expresiones numéricas al transformar las relaciones entre los datos y condiciones de un problema a una expresión numérica (modelo) que reproduzca las relaciones entre estos (esta expresión se comporta como un sistema compuesto por números, operaciones y sus propiedades); comunica su comprensión sobre los números y las operaciones al expresar la comprensión de los conceptos numéricos, las operaciones y propiedades, las unidades de medida, las relaciones que establece entre ellos (usando lenguaje numérico y diversas representaciones, así como leer sus representaciones e información con contenido numérico); usa estrategias, procedimientos de estimación y cálculo al seleccionar, adaptar, combinar o crear una variedad de estrategias, procedimientos como el cálculo mental y escrito, la estimación, la aproximación y medición, comparar cantidades y emplear diversos recursos; argumenta afirmaciones sobre las relaciones numéricas y las operaciones al elaborar afirmaciones sobre las posibles relaciones entre números naturales, enteros, racionales, reales, sus operaciones y propiedades (basado en comparaciones y experiencias en las que induce propiedades a partir de casos particulares) así como explicarlas con analogías, justificarlas, validarlas o refutarlas con ejemplos y contraejemplos”. 


\section{Metodología}

Se aplicaron los métodos científico, descriptivo, analítico-sintético; experimental y estadístico. Hernández y otros (2018) manifiestan que "los métodos empíricos de investigación pretenden estudiar las características fundamentales y las relaciones esenciales del objeto que son accesibles a la percepción sensorial". Se utilizó un diseño cuasi experimental. De acuerdo a Sánchez, Reyes \& Mejía (2018) es un "modelo o esquema que adopta el investigador para establecer un mejor control de las variables en estudio". Se trató de un estudio aplicativo, a un nivel explicativo, ya que nos permitió manipular la variable dependiente. La población fue conformada por 210 estudiantes y la muestra por 62 discentes del nivel secundaria, correspondiente al primer año de secundaria de una institución educativa de Lima metropolitana. Se administró una prueba pedagógica de entrada y salida, con características parecidas a la Prueba de la Evaluación Censal de Estudiantes que promueve el Ministerio de Educación del Perú. En cuanto a la validez del instrumento, se realizó por juicio de expertos. Los datos se analizaron utilizando el programa SPSS Para la comprobación de hipótesis se realizó mediante la prueba " $T$ ".

\section{$\underline{\text { Resultados }}$}

\section{Tabla 1}

Distribución de frecuencias de la prueba de entrada del Grupo Experimental

\begin{tabular}{|c|c|c|c|c|c|}
\hline \multicolumn{6}{|c|}{ Pe_ge } \\
\hline & & Frecuencia & Porcentaje & $\begin{array}{c}\text { Porcentaje } \\
\text { válido }\end{array}$ & $\begin{array}{l}\text { Porcentaje } \\
\text { acumulado }\end{array}$ \\
\hline \multirow[t]{8}{*}{$\overline{\text { Válido }}$} & 9 & 1 & 3,2 & 3,2 & 3,2 \\
\hline & 10 & 4 & 12,9 & 12,9 & 16,1 \\
\hline & 11 & 7 & 22,6 & 22,6 & 38,7 \\
\hline & 12 & 10 & 32,3 & 32,3 & 71,0 \\
\hline & 13 & 5 & 16,1 & 16,1 & 87,1 \\
\hline & 14 & 3 & 9,7 & 9,7 & 96,8 \\
\hline & 15 & 1 & 3,2 & 3,2 & 100,0 \\
\hline & Total & 31 & 100,0 & 100,0 & \\
\hline
\end{tabular}

Nota: Porcentaje de calificativos de la prueba de entrada del grupo experimental Fuente: Elaboración propia 


\section{Figura 1}

Resultados de la prueba de entrada del grupo experimental

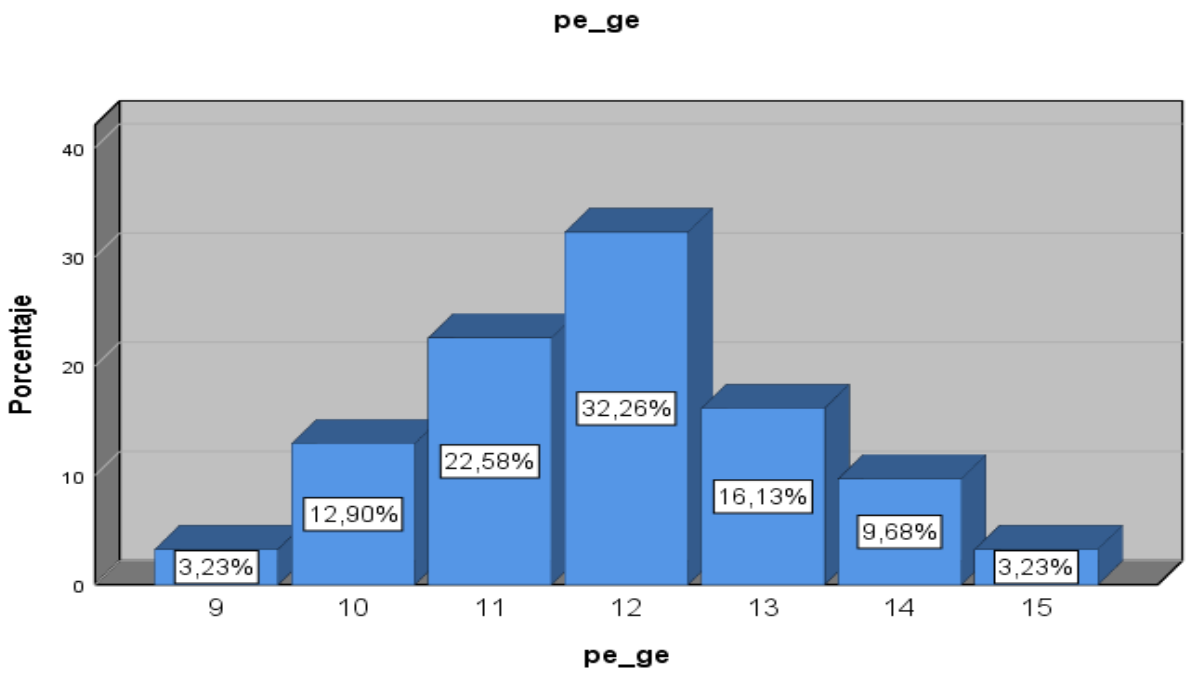

Nota: Porcentaje de calificativos de la prueba de entrada del grupo experimental Fuente: Elaboración propia

De acuerdo a los datos tabulados se observa que 1 estudiante alcanzó el puntaje de 9 y 15 puntos respectivamente (3,2\%), 4 estudiantes lograron 10 puntos (12,9\%), 7 alcanzaron 11 puntos (22,6), 10 sacaron 12 puntos (32,3\%), 5 alcanzaron 13 puntos $(16,1 \%)$ y 3 estudiantes lograron 14 puntos $(9,7 \%)$.

\section{Tabla 2}

Distribución de frecuencias de la prueba de entrada del Grupo Control

\begin{tabular}{llrrrr}
\multicolumn{7}{c}{ pe_gc } \\
\hline \hline Válido & \multicolumn{1}{c}{ Frecuencia } & Porcentaje & $\begin{array}{c}\text { Porcentaje } \\
\text { válido }\end{array}$ & $\begin{array}{c}\text { Porcentaje } \\
\text { acumulado }\end{array}$ \\
& 11 & 4 & 12,9 & 12,9 & 12,9 \\
& 12 & 12 & 38,7 & 38,7 & 51,6 \\
& 13 & 8 & 25,8 & 25,8 & 77,4 \\
& 14 & 4 & 12,9 & 12,9 & 90,3 \\
& 15 & 2 & 6,5 & 6,5 & 96,8 \\
& 17 & 1 & 3,2 & 3,2 & 100,0 \\
& Total & 31 & 100,0 & 100,0 & \\
\hline \hline
\end{tabular}

Nota: Porcentaje de calificativos de la prueba de entrada del grupo control Fuente: Elaboración propia 


\section{Figura 2}

Resultados de la prueba de entrada del grupo control

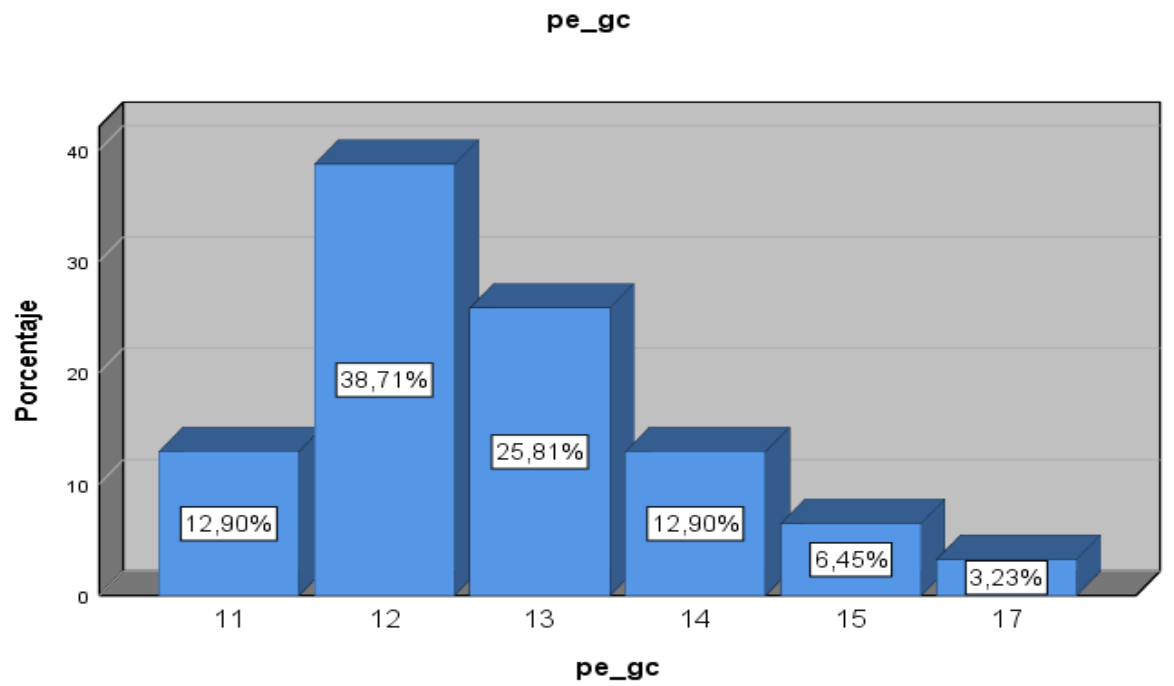

Nota: Porcentaje de calificativos de la prueba de entrada del grupo experimental Fuente: Elaboración propia

De acuerdo a los datos tabulados se observa 4 estudiantes alcanzaron el puntaje de 11 y 14 puntos respectivamente (12,9\%), 12 estudiantes lograron 12 puntos (38,7\%), 8 alcanzaron 13 puntos $(25,8), 2$ sacaron 15 puntos $(6,5 \%)$ y 1 estudiante logró 17 puntos $(3,2 \%)$.

Tabla 3

Distribución de frecuencias de la prueba de salida del Grupo Experimental

\begin{tabular}{rrrrrr}
\multicolumn{7}{c}{ ps_ge } \\
\hline \hline Válido & Frecuencia & Porcentaje & $\begin{array}{c}\text { Porcentaje } \\
\text { válido }\end{array}$ & $\begin{array}{c}\text { Porcentaje } \\
\text { acumulado }\end{array}$ \\
& 13 & 1 & 3,2 & 3,2 & 3,2 \\
& 15 & 7 & 22,6 & 22,6 & 25,8 \\
& 16 & 7 & 22,6 & 22,6 & 48,4 \\
17 & 7 & 22,6 & 22,6 & 71,0 \\
& 18 & 4 & 12,9 & 12,9 & 83,9 \\
19 & 3 & 9,7 & 9,7 & 93,5 \\
& 20 & 6,5 & 6,5 & 100,0 \\
& Total & 31 & 100,0 & 100,0 & \\
\hline \hline
\end{tabular}


Nota: Porcentaje de calificativos de la prueba de salida del grupo experimental Fuente: Elaboración propia

\section{Figura 3}

Resultados de la prueba de salida del grupo experimental

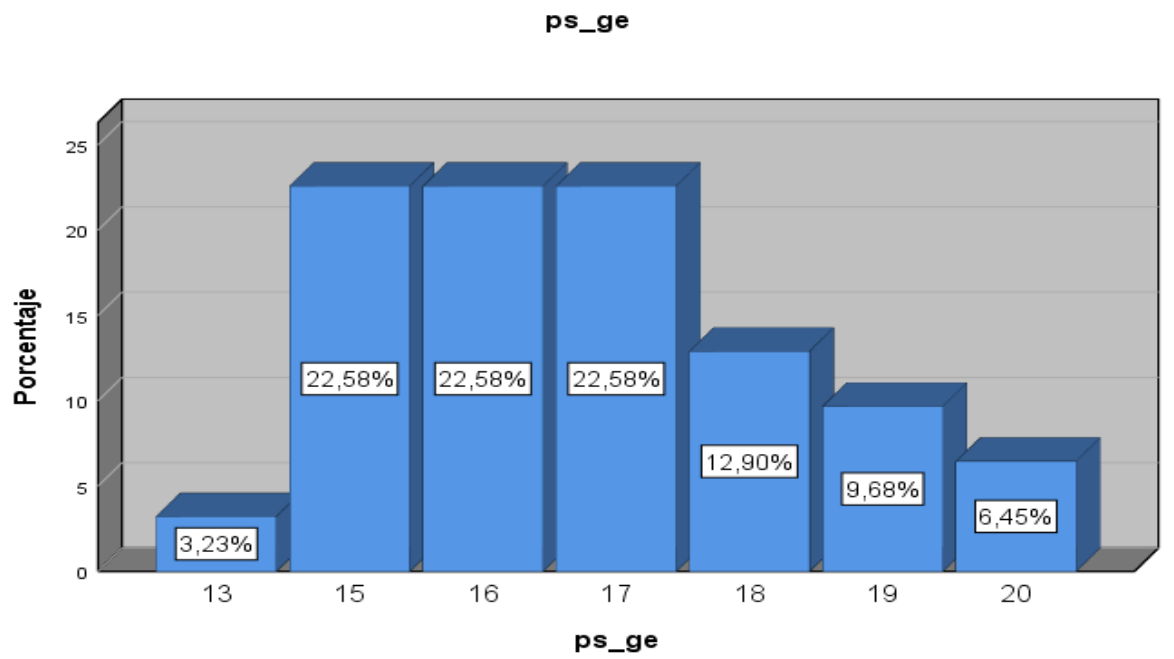

Nota: Porcentaje de calificativos de la prueba de entrada del grupo experimental Fuente: Elaboración propia

De acuerdo a los datos tabulados se observa 1 estudiante alcanzó el puntaje de 13 punto (3,2\%); 7 estudiantes lograron 15, 16 y 17 puntos respectivamente (22,6\%); 4 alcanzaron 18 puntos (12,9); 3 sacaron 19 puntos (9,7\%); y 2 estudiantes lograron 20 puntos.

\section{Tabla 4}

Distribución de frecuencias de la prueba de salida del Grupo Control

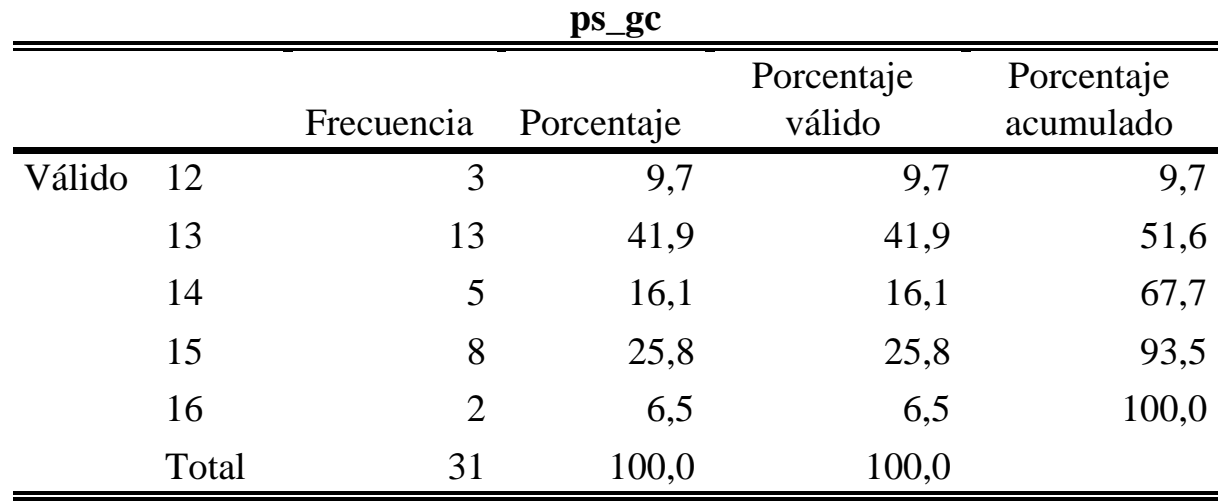

Nota: Porcentaje de calificativos de la prueba de salida del grupo control Fuente: Elaboración propia 


\section{Figura 4}

Resultados de la prueba de salida del grupo control

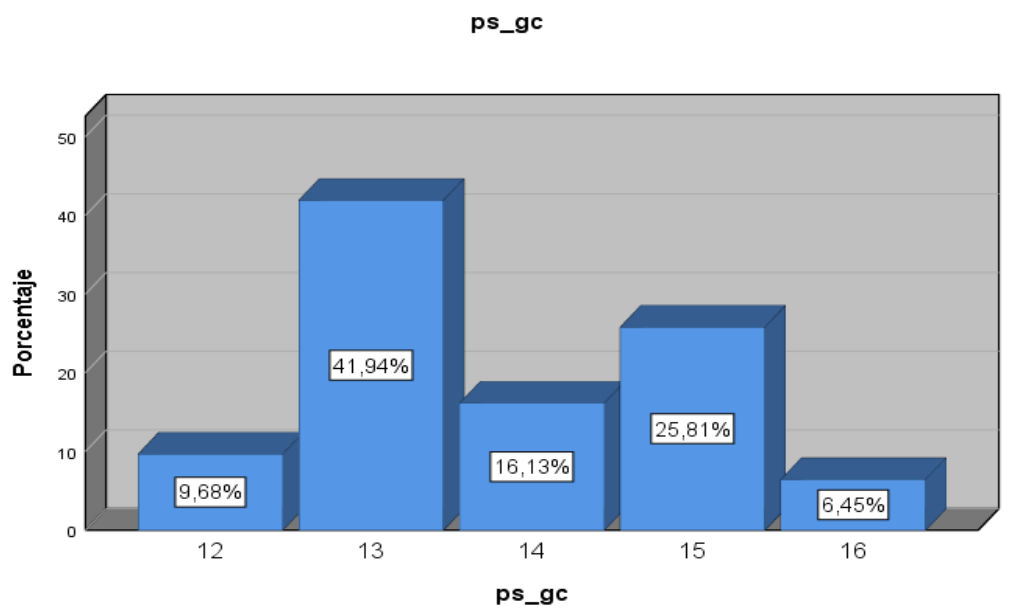

Nota: Porcentaje de calificativos de la prueba de entrada del grupo experimental Fuente: Elaboración propia

De acuerdo a los datos tabulados se observa 3 estudiantes alcanzó el puntaje de 12 punto $(9,7 \%), 13$ estudiantes lograron 13 puntos (41,9\%), 5 alcanzaron 14 puntos $(16,1 \%), 8$ sacaron 15 puntos $(25,8 \%)$ y 2 estudiantes lograron 16 puntos.

\section{Tabla 5}

Prueba de muestras independientes

Prueba de muestras independientes

\begin{tabular}{|c|c|c|c|c|c|c|c|c|c|}
\hline & & \multicolumn{4}{|c|}{$\begin{array}{c}\text { Prueba de Levene } \\
\text { de igualdad de } \\
\text { varianzas }\end{array}$} & \multicolumn{4}{|c|}{ prueba "t" para la igualdad de medias } \\
\hline & & \multirow[b]{2}{*}{$\mathrm{F}$} & \multirow[b]{2}{*}{ Sig. } & \multirow[b]{2}{*}{$\mathrm{t}$} & \multirow[b]{2}{*}{$\mathrm{gl}$} & \multirow{2}{*}{$\begin{array}{c}\text { Sig. } \\
\text { (bilateral) }\end{array}$} & \multirow{2}{*}{$\begin{array}{l}\text { Diferencia } \\
\text { de medias }\end{array}$} & \multirow{2}{*}{$\begin{array}{l}\text { Diferencia } \\
\text { de error } \\
\text { estándar }\end{array}$} & $\begin{array}{c}95 \% \text { de intervalo } \\
\text { de confianza de la } \\
\text { diferencia }\end{array}$ \\
\hline & & & & & & & & & Inferior Superior \\
\hline p_salida & $\begin{array}{l}\text { Se asumen } \\
\text { varianzas } \\
\text { iguales. }\end{array}$ & 2,952 &, 091 & 8,111 & 60 &, 000 & 2,93548 & ,36190 & $2,21157 \quad 3,65940$ \\
\hline
\end{tabular}


Nota: Porcentaje de calificativos de la prueba de entrada del grupo experimental Fuente: Elaboración propia

\section{Discusión}

De acuerdo a los resultados de las muestras independientes, como $\mathrm{p}<0,05$ se acepta la hipótesis alterna, concluyendo que los círculos de interaprendizaje tienen efectos significativos para incrementar "Logro Satisfactorio" en la competencia resolución de problemas de cantidad en estudiantes de primer año de secundaria, tal como se evidencia en las tablas 1 y 3 .

Estudios encontrados coinciden con los resultados presentados como Vigo Romero (2018) cuando dicen que "en la gestión curricular permitirá organizar de manera eficaz el proceso de planificación del currículo y de manera pertinente elaborar las programaciones, unidades y sesiones de aprendizajes, teniendo en cuenta las necesidades y sus estilos de aprendizaje de los estudiantes, con la finalidad de realizar un proyecto de tal manera que se enfoque el trabajo en equipo y colaborativo para consolidar una educación de calidad que permita la concreción de los aprendizajes fundamentales en los estudiantes”.

A su vez, Membrillo Cabrera (2018) al manifestar que "la implementación de la plataforma virtual Chamilo influyó de manera positiva en los círculos de interaprendizaje colaborativos de la I.E. San Ramón - La Recoleta - Cajamarca, trayendo consigo la optimización de tiempo y la posibilidad de debatir uno o más temas de manera simultánea" y Cornejo Turpo (2018) sostienen que "por otro lado existe correlación media entre la gestión pedagógica de los grupos de interaprendizaje y el trabajo docente con un coeficiente que equivale a 0,485 ; así como existe correlación entre la gestión pedagógica de los directivos y los grupos de interaprendizaje es media alta que corresponde a un nivel de 0,713 ; finalmente decimos que existe correlación entre la gestión pedagógica de los docentes y la gestión pedagógica de los directivos: es media alta que equivale a 0,822 (a un nivel de significancia del $5 \%$ en cada una de ellas)". 
También, Cabezas García (2016) manifiesta que "el estudio realizado permitió concluir que en la resolución de problemas una gran proporción (55.0\%) de estudiantes del quinto grado de primaria de la institución educativa N. ${ }^{\circ} 1230$ Viña Alta, La Molina, 2016, se situó en el nivel (en inicio) como elemento predominante entre ellos; lo cual deja entrever serias debilidades, limitaciones y dificultades en los estudiantes a la hora de la resolución de los problemas matemáticos". Fuentes López, Páez Gomez, \& Prieto (2019) proponen que "es importante trabajar la comprensión de textos, la estructura de la pregunta y el contexto de las situaciones planteadas desde todas las áreas del conocimiento desde el nivel de preescolar, dado a que ello incide en cómo el estudiante comprende o no las situaciones planteadas para adquirir el proceso en la resolución de problemas que se les planteen de acuerdo al nivel de complejidad".

Del mismo modo Añaños Flores \& Asencios Gonzáles (2018) concluyen que "se demostró que la resolución de problemas influye significativamente en el aprendizaje de la matemática en los estudiantes del cuarto grado. Los resultados demostraron que la resolución de problemas influye muy significativamente $(* * p<.01)$ en el aprendizaje de matemática". Domínguez Osorio y Espinoza Santiago (2019) dan a conocer que "una vez aplicado el Pretest, se realizaron las actividades de intervención en el grupo A, basadas en el método heurístico. Ello permitió potenciar la resolución de problemas matemáticos, en contraste con los resultados del grupo control. Esto confirma la hipótesis planteada y se evidencia en los resultados de esta investigación. La relevancia de este proyecto ha de tomarse como un modelo para futuras investigaciones en las distintas instituciones educativas locales, nacionales e internaciones".

De igual manera, Albán Alcívar (2018) plantean que "como resultados se identificaron las principales estrategias utilizadas para la resolución de problemas: repetición lectora, subrayado, lectoescritura, manipulación estratégica de incógnitas y datos informativos, esquematización procesal, empleo de cálculo aritmético y graficación. La incidencia de las estrategias determinó que el promedio mayor corresponde al grupo de mayor rendimiento académico para la resolución de los problemas matemáticos y el promedio más bajo está 
relacionado con el uso mínimo de estrategias en cada una de las etapas de resolución de un problema".

Y también, Paredes Lozada (2019) expresa que "el 35\% de los profesores aplican bien las estrategias metodológicas para resolver problemas y, en respuesta, se tiene que el $24 \%$ de los alumnos logran un buen desarrollo de sus capacidades matemáticas. El 54\% de los estudiantes desarrollan sus capacidades matemáticas de manera irregular y de bajo nivel. Los profesores de la I.E. 1137 José Antonio Encinas, a pesar que el 80 \% ha recibido alguna capacitación en la aplicación de estrategias metodológicas heurísticas de G. Pólya, el 33\% lo hace de manera incompleta y el $32 \%$ no aplica estas estrategias".

Finalmente, Zona-López \& Giraldo-Márquez (2017) consideran que “el análisis de las frecuencias en las respuestas de los estudiantes, según los diferentes niveles de resolución de problemas, muestra cierta tendencia al empleo de niveles más exigentes a medida que la intervención de aula avanza. Es necesario replantear intervenciones a mediano y largo plazo, en donde se incorporen y desarrollen problemas auténticos en las aulas de clase, problemas que permiten generar múltiples respuestas, respuestas abiertas justificadas con razones de validez que permiten comprender los fenómenos en forma abstracta”.

\section{Conclusiones}

- Los círculos de interaprendizaje tienen efectos significativos para incrementar "Logro Satisfactorio" en la competencia resolución de problemas de cantidad en estudiantes de primer año de secundaria.

- Los círculos de interaprendizaje tienen efectos significativos para incrementar "Logro Satisfactorio" en la competencia resolución de problemas de cantidad en la capacidad traduce cantidades a expresiones numéricas de estudiantes de primer año de secundaria.

- Los círculos de interaprendizaje tienen efectos significativos para incrementar "Logro Satisfactorio" en la competencia resolución de problemas de cantidad en la capacidad comunica su comprensión sobre los números y las operaciones de estudiantes de primer año de secundaria. 
- Los círculos de interaprendizaje tienen efectos significativos para incrementar "Logro Satisfactorio" en la competencia resolución de problemas de cantidad en la capacidad usa estrategias y procedimientos de estimación y cálculo de estudiantes de primer año de secundaria.

- Los círculos de interaprendizaje tienen efectos significativos para incrementar "Logro Satisfactorio" en la competencia resolución de problemas de cantidad en la capacidad argumenta afirmaciones sobre las relaciones numéricas y las operaciones de estudiantes de primer año de secundaria.

\section{$\underline{\text { Referencias }}$}

Albán J. (2018). Estrategias que utilizan los estudiantes para la resolución de un problema matemático y su incidencia en el rendimiento académico. Tesis para optar el título de Magíster en Docencia de las Matemáticas, Universidad de Cuenca, Facultad de Filosofía, letras y ciencias de la Educación, Cuenca. Obtenido de https://n9.cl/0898h

Añaños M., \& Asencios Gonzáles, H. (2018). La resolución de problemas en el aprendizaje de matemática en estudiantes del cuarto grado de educación secundaria de la institución educativa "Manuel González Prada” de Huari - 2016. Tesis para optar el grado de Magíster, Universidad Católica Sedes Sapientiae, Escuela de posgrado. Obtenido de https://n9.cl/bm0j

Cabezas C. (2016). Resolución de problemas en los estudiantes del quinto grado de primaria de la institución educativa n. 1230 Viña Alta, La Molina, 2016. Universidad César Vallejo, Facultad de Educación e Idiomas, Lima. Obtenido de https://repositorio.ucv.edu.pe/bitstream/handle/20.500.12692/17725/Cabezas_GCL. pdf? sequence $=1 \&$ is Allowed $=\mathrm{y}$

Cornejo M. (2018). Gestión pedagógica del docente y grupos de interaprendizaje en las instituciones educativas de nivel de educación primaria. Tesis para optar el grado académico de Doctor en Educación, Universidad Andina Néstor Cáceres Velásquez, Escuela de posgrado. Obtenido de https://n9.cl/ptubp

Domínguez L., \& Espinoza Santiago, B. (2019). Potenciar la resolución de problemas matemáticos desarrollando habilidades de pensamiento desde una mirada heurística. Trabajo de grad para optar al título de Magíster en Educación, Universidad de la Costa, Facutad de Humanidades, Barranquilla. Obtenido de https://n9.cl/6damw

Fuentes, C., Páez Gomez, P., \& Prieto, D. (2019). Dificultades de la resolución de problemas matemáticos de estudiantes de grado 501 Colegio Floresta Sur, Sede n, jornada tarde, Localidad de Kénedy. Trabajo para obtener el título de Magíster, 
Universidad Cooperativa de Colombia, Facultad de Educación. Obtenido de https://n9.cl/2mm5

Hernández, A., Ramos, M., Placencia, B., Indacochea, B., Quimis, A., \& Moreno, L. (2018). Metodología de la investigación científica. Manabí: Editorial Área de Innovación y Desarrollo SL.

Membrillo, C. (2018). Implementación de una plataforma virtual y su influencia en los círculos de interaprendizaje colaborativos en la I.E. San Ramón - La RecoletaCajamarca - 2017. Tesis para optar el título profesional de Ingeniero Informático y de Sistemas, Universidad Privada Antonio Guillermo Urrelo, Escuela Profesional de Ingeniería Informática y Sistemas, Cajamarca. Obtenido de https://n9.cl/4gxgi

Ministerio de Educación del Perú. (2016). Currículo Nacional de la Educación Básica. Lima: MINEDU.

Ministerio de Educación. (s/a). Manual para los grupos de interaprendizaje - GIA. Lima: Universidad Nacional Mayor de San Marcos. Obtenido de https://n9.cl/fje8r

Organización de las Naciones Unidas para la Educación, la Ciencia y la Cultura (UNESCO). (2019). La formación docente en servicio en el Perú: Proceso de diseño de políticas y generación de evidencias. Lima: UNESCO. Obtenido de https://n9.cl/4awb1

Paredes, J. (2019). Estrategia metodológica para resolver problemas y el desarrollo de capacidades matemáticas en estudiantes de primaria de la institución educativa 1137 “José Antonio Encinas”. Tesis para optar el título de licenciado en Educación, Universidad San Martín de Porres, Escuela profesional de educación. Obtenido de https://n9.cl/agxyf

Proyecto Regional Andino Perú-Bolivia. (25 de Julio de 2010). Ancash: Círculos de interaprendizaje, aprendiendo desde el colectivo. Obtenido de Proyecto Regional Andino Perú-Bolivia: https://n9.cl/ny6zt

Sánchez, H., Reyes, C., \& Mejía, K. (2018). Manual de términos de investigación científica, tecnológica y humanista. Lima: Universidad Ricardo Palma.

Toribio V., \& Guerrero Ortiz, L. (2017). Monitoreo, acompañamiento y evaluación de la práctica docente. Lima: Programa Directivos Educan del Ministerio de Educación.

Vigo J. (2018). Los círculos de interaprendizaje como estrategia para mejorar la comprensión de textos escritos en la I.E. Santa Úrsula de Hualcán. Trabajo Académico para optar el Título Profesional de Segunda Especialidad, Universidad San Ignacio de Loyola, Escuela de posgrado. Obtenido de https://n9.cl/46bqw 
Zona J., \& Giraldo-Márquez, J. (Julio-Diciembre de 2017). Resolución de problemas:

escenario del pensamiento crítico en la didáctica de las ciencias. Revista

Latinoamericana de Estudios Educativos, 13(2), 122-150. Obtenido de

https://www.redalyc.org/pdf/1341/134154501008.pdf

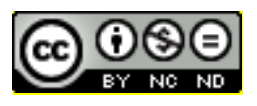

Círculos de interaprendizaje para incrementar logro satisfactorio en resolución de problemas de cantidad en estudiantes de primer año de secundaria (Juan Carlos Cárdenas - Valverde) Por $\underline{\text { Revista Sendas se encuentra bajo una Licencia Creative Commons-No Comercial-Sin Derivadas }}$

3.0 Uported. 